\title{
Erratum: More efficient formulas for efficiency correction of cumulants and effect of using averaged efficiency [Phys. Rev. C 95, 064912 (2017)]
}

Toshihiro Nonaka $\odot$, Masakiyo Kitazawa $\odot$, and ShinIchi Esumi $\odot$

(Q) (Received 8 December 2020; published 8 February 2021)

DOI: 10.1103/PhysRevC.103.029901

There is a typographical error in Eq. (70) in the original paper. Equation (70) should read

$$
\begin{aligned}
\left\langle Q_{(x)}^{2} Q_{(y)}\right\rangle_{\mathrm{c}}= & \left\langle q_{(1,0,1)}^{2} q_{(0,1,1)}\right\rangle_{\mathrm{c}}+2\left\langle q_{(1,0,1)} q_{(1,1,1)}\right\rangle_{\mathrm{c}}-2\left\langle q_{(1,0,1)} q_{(1,1,2)}\right\rangle_{\mathrm{c}}+\left\langle q_{(0,1,1)} q_{(2,0,1)}\right\rangle_{\mathrm{c}} \\
& -\left\langle q_{(0,1,1)} q_{(2,0,2)}\right\rangle_{\mathrm{c}}+\left\langle q_{(2,1,1)}\right\rangle_{\mathrm{c}}-3\left\langle q_{(2,1,2)}\right\rangle_{\mathrm{c}}+2\left\langle q_{(2,1,3)}\right\rangle_{\mathrm{c}},
\end{aligned}
$$

where the multiplication factor " 2 " for the last term on the right-hand side was missing in the original paper. This typographical error does not affect any of the results and conclusions. 